\title{
CUNI Neural ASR with Phoneme-Level Intermediate Step for Non-Native SLT at IWSLT 2020
}

\author{
Peter Polák and Sangeet Sagar and Dominik Macháček and Ondřej Bojar \\ Charles University \\ Faculty of Mathematics and Physics \\ Institute of Formal and Applied Linguistics \\ \{polak, sagar,machacek, bojar\}@ufal.mff.cuni.cz
}

\begin{abstract}
In this paper, we present our submission to the Non-Native Speech Translation Task for IWSLT 2020. Our main contribution is a proposed speech recognition pipeline that consists of an acoustic model and a phoneme-tographeme model. As an intermediate representation, we utilize phonemes. We demonstrate that the proposed pipeline surpasses commercially used automatic speech recognition (ASR) and submit it into the ASR track. We complement this ASR with off-the-shelf MT systems to take part also in the speech translation track.
\end{abstract}

\section{Introduction}

This paper describes our submission to Non-Native Speech Translation Task in IWSLT 2020 (Ansari et al., 2020). We participate in two sub-tracks: offline speech recognition and offline speech translation from English into Czech and German.

We focus on the speech recognition, proposing a robust pipeline consisting of two components an acoustic model recognizing phonemes, and a phoneme-to-grapheme translation model, see Figure 1 . We decided to use phonemes as the intermediate representation between the acoustic and the translation model because we believe that conventional grapheme representation is too constrained with complicated rules of mapping speech to a transcript. This issue becomes immense when dealing with dialects and non-native speakers.

Both models used in our pipeline are end-toend deep neural networks, Jasper (Li et al., 2019) for the acoustic model and Transformer (Vaswani et al., 2017) for the phoneme-to-grapheme translation model.

For punctuating, truecasing, segmenting and translation into Czech and German, we use offthe-shelf systems provided by ELITR project.

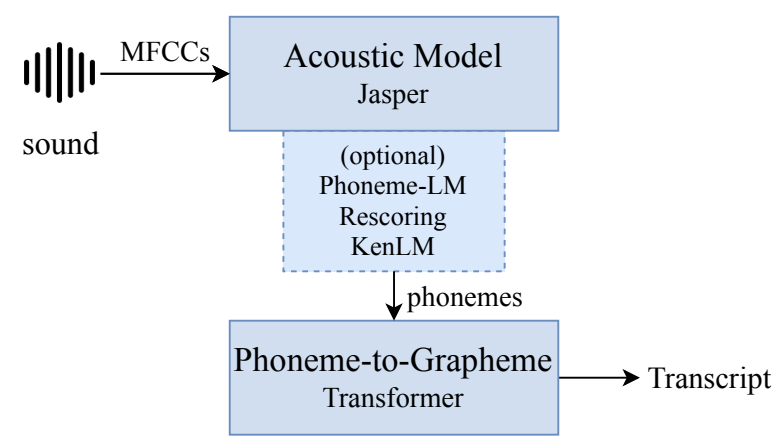

Figure 1: The architecture of proposed model.

The paper is organized as follows: Section 2 reviews related work. In Sections 3 and 4 we describe models for our speech recognition pipeline and their training. In Section 5, we describe the punctuator, truecasor and segmenter, and machine translation into Czech and German in Section 6. We summarize our submissions in Section 7 and conclude in Section 8.

\section{Related Work}

This section reviews the related work.

\subsection{Phonemes and Acoustic Models}

Phones and phonemes are well-established modelling units in ASR. They have been used since the beginning of the technology in 1950s (Juang and Rabiner, 2005), for an empirical comparison of different linguistic units for sound representation, see Riley and Ljolje (1992).

An important work popularizing neural networks in ASR to phonemes is Waibel et al. (1989). This work proposes using a time-delayed neural network (TDNN) to model acoustic-phonetic features and the temporal relationship between them. The authors demonstrate that the proposed TDNN can learn shift-invariant internal abstraction of speech and use it to make a robust final decision. 
Salesky et al. (2019) suggest using of phonemebased ASR in speech translation. Their end-to-end speech translation pipeline first obtains phoneme alignment using the deep neural network hidden Markov models (DNN-HMM) system and then averages feature vectors with the same phoneme for consecutive frames. Phonemes outputted by DNNHMM then serve as input features for speech translation.

\subsection{Phoneme-to-Grapheme Models}

In most past studies that included a separate phoneme-to-grapheme (P2G) translation component into the ASR, the phoneme representation was used only for out-of-vocabulary (OOV) words, see, e.g. Decadt et al. (2001); Horndasch et al. (2006); Basson and Davel (2013).

Decadt et al. (2001) apply phoneme-tographeme to enhance the readability of OOV output in Dutch speech recognition. In their setup, the ASR outputs standard (orthographic) text for known words. For OOVs, phonemes are outputted. Because the phonemes are unreadable for most users, the authors translate phonemes using memory-based learning. The word error rate of this improved setup of Dutch ASR was actually higher than the baseline, on the other hand, the output was better readable for an untrained person. They report that $41 \%$ of words were transcribed with at most one error, and $62 \%$ have only two errors. Furthermore, most of the incorrectly transcribed words do not exist in Dutch.

Horndasch et al. (2006) introduce a data-driven approach called MASSIVE. Their main objective is to find appropriate orthographic representations for dictated Internet search queries. Their system iteratively refines sequences of symbol pairs in different alphabets. In the first step, they find the best phoneme-grapheme alignment using the expectation-maximization algorithm. In the second step, they cluster neighbouring symbols together to account for insertions. Finally, $n$-gram probabilities of symbol pairs are learned. During the inference, the input string is split into individual symbols. All possible symbol pairs are generated for each symbol, and the best sequences are selected in a beam search.

\subsection{Error Correction in ASR}

Hrinchuk et al. (2019) deal with the correction of errors in ASR by introducing Transformer postprocessing. The authors first train an ensemble of
10 ASR models. Using these models, they collect "ASR corrupted" data. Subsequently, they train a Transformer on this data where the "ASR corrupted" text serves as the source and the original true transcripts as the target. In their best setup, they utilize transfer learning. They use BERT (Devlin et al., 2018), a masked language model consisting only of Transformer encoder, and initialize both encoder and decoder of their Transformer correction model with BERT's weights.

\subsection{Online ASR Services}

We compare our work with Google Cloud Speechto-Text API ${ }^{1}$ and Microsoft Azure Speech to Text. ${ }^{2}$ Both of these services provide publicly available APIs for transcribing audio recordings.

\section{Neural ASR with Phoneme-Level Intermediate Step}

Our main idea is to couple an end-to-end acoustic model with a specialized "translation" model, which translates phonemes to graphemes and corrects the ASR errors.

The motivation for the translation step is that the translation model can exploit larger context than a basic convolutional acoustic model. Furthermore, we can utilize considerably larger non-speech corpora to train this part of the pipeline.

\subsection{Acoustic Model}

For our acoustic model, we use the Jasper (Li et al., 2019) convolutional neural architecture in the variant of Jasper DR 10x5 variant, as described in the original paper. It is implemented within the NeMo library (Kuchaiev et al., 2019).

For training, we use approximatelly 1000 hours of speech data from LibriSpeech (Panayotov et al., 2015) and 1000 hours of Common Voice ${ }^{3}$. Because we want the model to produce phonemes and not graphemes, which are available in the training corpora, we converted the transcript to IPA phonemes using the phonemizer ${ }^{4}$ tool.

To speed-up the training process, we initialize our English sound-to-phoneme Jasper model with

\footnotetext{
${ }^{1}$ https://cloud.google.com/ speech-to-text

${ }^{2}$ https: / / azure.microsoft.com/ en-us/services/cognitive-services/ speech-to-text/

${ }^{3}$ https://voice.mozilla.org/en

${ }^{4}$ https://github.com/bootphon/ phonemizer
} 


\begin{tabular}{cc|cc} 
Type & Corpus & Adapt. & Full training \\
\hline \multirow{2}{*}{ dev } & LS Clean & 46.07 & 3.84 \\
& CV & 54.69 & 11.86 \\
\hline \multirow{3}{*}{ test } & LS Clean & - & $4.18 / 4.48 \dagger / 3.58 \ddagger$ \\
& LS Other & - & $11.48 / 11.67 \dagger / 8.57 \ddagger$ \\
& CV & - & $10.21 / 10.47 \dagger / 6.46 \ddagger$
\end{tabular}

Table 1: Results in \% of Phoneme Word Error Rate (PWER) using greedy decoding (no mark), beam search $(\dagger)$ and beam search with language model $(\ddagger)$. The language model is trained on phonemized ASR training data. Note, PWER is not directly comparable to WER. "LS" LibriSpeech. "CV" Common Voice.

the available checkpoint of the standard sound-tographeme model. ${ }^{5}$. This seed model was trained on LibriSpeech, Mozilla Common Voice, WSJ, Fisher, and Switchboard corpora, which is beyond the set of corpora allowed for a constrained submission. The model yields word error rate (WER) of 3.69 $\%$ on LibriSpeech test-clean, and $10.49 \%$ on testother using greedy decoding.

For a smooth transition from the Latin alphabet to IPA, we start our training with an adaptation phase of 2,000 training steps. As the model's memory footprint is smaller during this phase, we increase the batch size to 64 (global batch size is 640). One thousand steps are warm-up; the maximal learning rate is 0.004 .

The full training takes ten epochs. The model memory requirements increase, therefore we reduce the batch size to 16 (global batch size is 160). We also reduce the learning rate to 0.001 .

Optionally, we include a phoneme-level language model, which re-scores the output of the acoustic model before the phoneme-to-grapheme translation, to achieve higher quality. Setups that use this component are further in this paper marked with " $1 \mathrm{~m}$ ".

Results of training after the Adaptation phase (the "Adaptation" column) and the Full training are in Table 1. Note that these scores are calculated on the reference transcript converted to phonemes using phonemizer. Token ambiguities thus change, and these scores are not comparable to standard grapheme WER.

The training is executed on 10 NVIDIA GTX 1080 Ti GPUs with 11 GB VRAM.

\footnotetext{
${ }^{5}$ https://ngc.nvidia.com/catalog/ models/nvidia:multidataset_jasper $10 \times 5 \mathrm{dr}$
}

\section{Phoneme-to-Grapheme Model}

We seek a model for translating transcripts written in phonemes into graphemes in the same language. Unlike the most studies reviewed in Section 2, we propose to use Transformer (Vaswani et al., 2017) architecture for phoneme-to-grapheme translation. We believe that Transformer is the best option for these tasks. Transformer has shown its potential in many NLP tasks. Most importantly, we consider its ability to learn the structure of a sentence, see e.g. Pham et al. (2019).

\subsection{Text Encoding Considerations}

We use Byte Pair Encoding (BPE) (Sennrich et al., 2016) for text encoding in our experiments. We use the implementation in YouTokenToMe ${ }^{6}$ library. It is fast and offers BPE-dropout (Provilkov et al., 2019) regularization technique.

First, we decided to use separate vocabularies for source and target sentences, because the source and target representations, IPA phonemes and English graphemes, have no substantial overlap.

There has been a quite intensive discussion about vocabulary size in neural machine translation (NMT) (Denkowski and Neubig, 2017; Gupta et al., 2019; Ding et al., 2019). All works agree that for low-resource translation tasks, it is better to apply smaller vocabulary sizes. For a high-resource task, it is convenient to use larger vocabulary. Our task, translation of phonemes into graphemes in the same language, differs from the previous works. Hence, we decided to experiment with vocabulary sizes. We also want to know whether we should train the sub-word units for source on clean data (phonemes without errors), or we should introduce ASR-like errors to these data.

We design the experiment as follows: we test character-level encoding and BPE vocabulary sizes of 128, 512, 2000,8000 and 32 000. Further, we test a clean data configuration, "corrupted" data (we collect transcripts from an ensemble of 10 ASR systems) and a "mixed" data - combination of the two previous.

Because of the data scarcity, we use Transformer Base configuration. We alter maximum sequence length to 1024 because for character-level, 128, and 512 BPE configurations, many sentences do not fit into the model. We train all models for 70000 steps on one GPU using the same batch size for all configurations: 12000 tokens. We set the learning rate

\footnotetext{
${ }^{6}$ https://github.com/VKCOM/YouTokenToMe
} 
to 0.04. As training data, we use "corrupted" ASR transcripts paired with true transcripts. We collect the data from an ensemble of 10 ASR models, yielding approximately 7 million sentence pairs. For the collection of ASR corrupted data, we used LibriSpeech and Common Voice datasets.

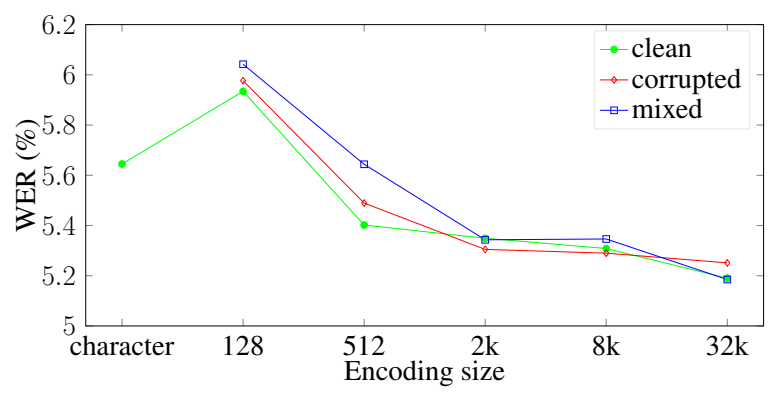

Figure 2: Results in \% of word error rate on the Common Voice test set.

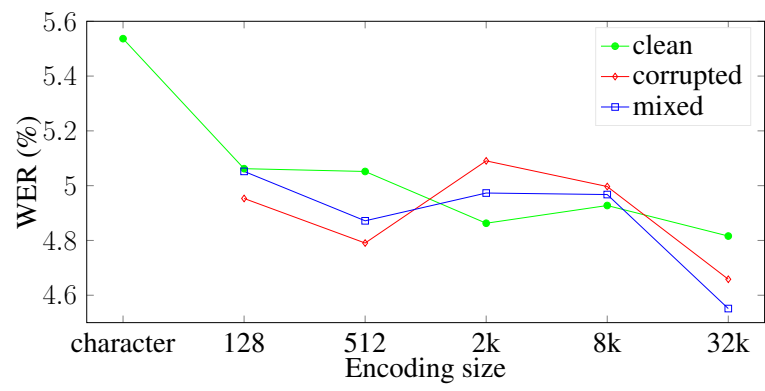

Figure 3: Results in \% of word error rate on the LibriSpeech test clean.

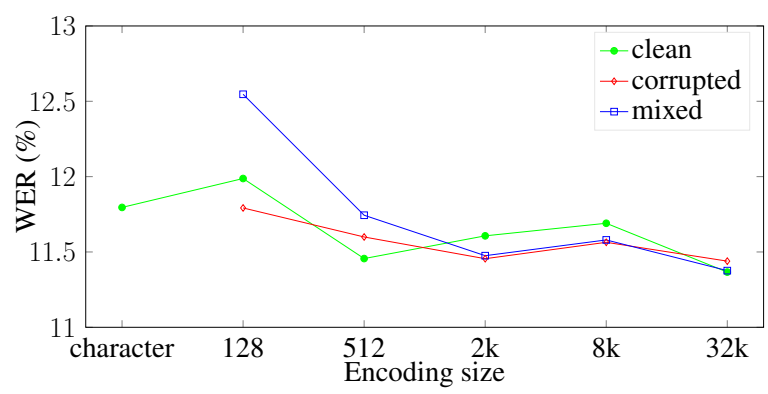

Figure 4: Results in \% of word error rate on the LibriSpeech test other.

Graphical comparison is in Figures 2 to 4.

BPE size Character-level encoding seems to be the worst or second-worst possible representation. For the Common Voice test set, it scores almost one percentage point of WER more compared to the best result (5.53 vs 4.55 ). Also, all other encodings performed almost half a percentage point better.
For both LibriSpeech test sets, it performed a bit better than BPE 128.

Generally, the results suggest a the larger the vocabulary, the lower WER. Among the different BPE sizes, we can recognize the 32000 vocabulary size has the best results systematically on all test sets.

Finally, we consider the following: a model can better learn from larger vocabulary sizes. First, a model does not have to learn low-level orthography extensively. Rather than memorizing characters (or other smaller units), it can focus on the whole sentence and how individual words interact. Second, a larger model can detect errors because of anomalies in the input encoding. Larger vocabularies produce a shorter representation. Corrupted word is more likely to be broken down to smaller pieces. When a model detects such a situation, it can, for example, decide the right target word based on context, rather than the suspicious word. Such anomaly will most likely not occur in the text encoded with small BPE.

Source of BPE training data For Common Voice, we observe some variation in performance. Best seems to be the "mixed" configuration. Somewhat worse is "corrupted" and the worst is "clean" version. In this case, we think the "mixed" is best as it has frequent enough "corrupted" words. This enables a model to learn to translate these corrupted words into the correct ones. Also, it knows enough other words, so it can adequately work with correct phonemes.

For other test sets, we observe almost no differences. Only "corrupted" configuration has slightly worse performance.

We conclude that the source of training data for BPE has almost no impact on the final result.

\subsection{Baseline Phoneme-to-Grapheme Model ("ass" Configuration)}

We decided to use Transformer Big configuration (as opposed to the initial experiment with BPE vocabularies). As we concluded in the previous part, we select BPE vocabulary size of 32000 , and the BPE encoding is trained on "clean" phonemized English part of Czeng 1.7 (Bojar et al., 2016) corpus.

First, we train a randomly initialized Transformer model. The source of the "translation" is the phonemized English Czeng and the target is the original English. 
We use six 16 GB GPUs for the training. We set the batch size to 6000 tokens, learning rate to 0.02 , warm-up steps to 16000 and total steps to 600000 . We manually abort the training after the convergence is reached (140000 steps in our case).

\subsection{Transfer from SLT ("asr_slt" Configuration)}

In standard NMT, the source text usually does not suffer from so many errors as in our setup. We address this "correction" need by training on artificially corrupted source side.

We initialize the Transformer encoder from our in-house speech translation model trained from English phonemes to Czech graphemes (described in Polák (2020)) and the decoder from a model for the opposite direction. Both of these initial models were trained on CzEng, with one side converted to phonemes using phonemizer.

These pre-trained parts of the model, the encoder and decoder need joint training to learn to operate with each other. We employ this training also to inject the capacity of correcting ASR output.

Specifically, we apply the jack-knife scheme to our ASR training data (LibriSpeech and Common Voice), training ten different ASR models, always leaving one-tenth of the training data aside. This one-tenth is recognized with the model, leading to the full speech corpus equipped not only with golden transcripts but also with ASR outputs. We call this an "ASR-corrupted" corpus.

Based on our experience from the experiment with BPE vocabularies, where the model easily over-fit to the sentences from ASR transcripts from speech corpora, we mix the corrupted and clean data with a 1:1 ratio. This is different from Hrinchuk et al. (2019) who use only the ASRcorrupted data to train. We then train the complete Transformer model from English phonemes to English graphemes with the same hyper-parameters as the baseline.

\subsection{Transfer from BERT ("asr_bert" Configuration)}

Finally, we use the pre-trained BERT (Devlin et al., 2018). Unlike Hrinchuk et al. (2019), we do not initialize both the encoder and decoder with the BERT. We initialize the encoder from the English-toCzech speech translation model (as in Section 4.3) because we need the model to process phonemes, not graphemes on the source side. The decoder is initialized from the BERT "large" to match the dimension of the Transformer encoder.

For this setup, we tried the same training procedure on half-noisy data as above. However, we were unable to obtain any reasonable performance (we got WER of $28 \%$ on LibriSpeech dev-other). We hypothesize this is due to the vast amount of weights that must be randomly initialized in the decoder: BERT is a Transformer encoder only. Hence it does not have the Encoder-Decoder attention layer which must be trained from scratch. During the training of the whole model with many randomly initialized weights, the initially trained weights from the BERT might depart too far from the optimum.

To overcome this issue, we use an analogous adaptation trick as for the training of the acoustic model. We freeze all weights initialized from seed models and train only the randomly initialized weights until convergence (the criterion was the loss on the validation dataset). This adaptation takes 13500 steps in our case. Subsequently, the training continues as in the previous case with one exception - we used only ASR corrupted data from LibriSpeech.

\subsection{ASR Results}

\begin{tabular}{l|rrr} 
& CV & LS clean & LS other \\
\hline asr (primary) & 9.72 & 4.87 & 11.67 \\
asr_lm & 7.00 & 4.63 & 10.25 \\
asr_slt & $\mathbf{3 . 2 6}$ & 5.10 & 11.75 \\
asr_slt_lm & 3.97 & 5.00 & 10.63 \\
bert & 12.93 & 4.13 & 10.21 \\
bert_lm $\dagger$ & 11.25 & $\mathbf{4 . 0 4}$ & $\mathbf{9 . 6 9}$
\end{tabular}

Table 2: Performance of the submitted models in terms of \% WER on the Common Voice test set (CV), and LibriSpeech (LS) clean and other test set. $\dagger$ not submitted due to time constraints. Best results in bold.

Table 2 reports the performance of our proposed systems on Common Voice test set and LibriSpeech test-clean and test-other.

The performance of "slt"-pretrained models is very good on Common Voice (CV), reaching WER of $3.26 \%$. However, we suspect that the model overfitted to $\mathrm{CV}$ texts. The corpus contains many speakers, but the set of underlying sentences is very limited, and our models can memorize them. The more realistic evaluation on the independent LibriSpeech other indicates that "asr_slt" is actually rather poor.

For the general domain, assessed by LibriSpeech 


\begin{tabular}{|c|c|c|c|c|c|c|c|c|c|c|}
\hline & \multirow{2}{*}{ AMIa } & \multirow{2}{*}{ AMIb } & \multirow{2}{*}{ AMIc } & \multirow{2}{*}{ AMId } & \multirow{2}{*}{ Teddy } & \multirow{2}{*}{ Autocentrum } & \multirow{2}{*}{ Audit } & \multicolumn{3}{|c|}{ Weightened } \\
\hline & & & & & & & & AMI & Rest & Total \\
\hline asr (primary) & 35.89 & 32.76 & 35.60 & 39.90 & 57.43 & 11.62 & 9.83 & 35.05 & 19.67 & 33.99 \\
\hline asr_lm $\_$ & 37.58 & 33.66 & 35.32 & 40.60 & 56.65 & 14.01 & 11.00 & 35.70 & 20.54 & 34.65 \\
\hline asr_slt & 36.73 & 33.22 & 35.70 & 39.69 & 56.87 & 10.93 & 10.22 & 35.37 & 19.66 & 34.28 \\
\hline asr_slt_lm $\dagger$ & 37.71 & 33.83 & 35.67 & 40.45 & 56.31 & 12.87 & 10.71 & 35.88 & 20.07 & 34.78 \\
\hline asr_bert & 36.69 & 33.82 & 36.50 & 39.63 & 56.76 & 12.87 & 9.60 & 35.85 & 19.64 & 34.72 \\
\hline asr_lm $\ddagger$ & 35.95 & 32.94 & 35.57 & 40.43 & 56.20 & 13.10 & 10.67 & 35.20 & 20.22 & 34.17 \\
\hline asr_slt_lm & 37.72 & 33.86 & 35.59 & 40.59 & 56.42 & 13.10 & 10.71 & 35.88 & 20.29 & 34.80 \\
\hline Micr & 53.72 & 52.62 & 56.67 & 58.58 & 87.82 & 39.64 & 24.22 & 54.80 & 39.75 & 53.76 \\
\hline Google & 51.52 & 49.47 & 53.11 & 56.88 & 61.01 & 14.12 & 17.47 & 51.87 & 25.33 & 50.03 \\
\hline
\end{tabular}

Table 3: Results in \% WER on IWSLT ASR development set. $†$ submitted without punctuation and segmentation. $\ddagger$ submitted with punctuation and segmentation after the deadline.

\begin{tabular}{l|cccc} 
& AMIa & Teddy & Autocentrum & Audit \\
\hline asr & 4.79 & 1.41 & 21.66 & 5.59 \\
asr_lm $\dagger$ & 2.80 & 1.57 & 12.53 & 1.84 \\
asr_lm $\dagger^{+*}$ & 2.86 & 1.57 & 12.79 & 1.93 \\
asr_slt & 4.52 & 1.48 & $\mathbf{2 2 . 0 2}$ & 5.56 \\
asr_slt_lm $\dagger$ & 3.19 & 1.55 & 8.85 & 1.81 \\
asr_slt_lm $\dagger^{*}$ & 3.26 & 1.55 & 9.32 & 1.88 \\
asr_bert & $\mathbf{6 . 0 8}$ & 1.41 & 19.01 & $\mathbf{5 . 7 9}$ \\
asr_lm $\ddagger$ & 3.92 & 5.65 & 21.65 & 5.24 \\
asr_slt_lm $\ddagger$ & 4.01 & $\mathbf{6 . 0 8}$ & 21.50 & 5.02 \\
\hline Gold & 21.09 & 54.77 & 42.52 & 9.03
\end{tabular}

Table 4: Czech BLEU scores on the IWSLT development set. $\dagger$ submitted without punctuation and segmentation. $\ddagger$ submitted with punctuation and segmentation after the deadline. * lower case BLEU.

clean, we would choose the BERT-pretrained model with phoneme LM rescoring. This model was unfortunately trained too late, so we did not include it in our submission.

The Non-Native Task setting is very specific, and we carefully examine the performance on the IWSLT development (Table 3). The performance varies considerably, but the baseline setup ("asr") perform well on average, and it is also not much worse than the best system on the particular files, e.g. 9.83 on the Audit file compared to "asr_bert" which wins there with 9.60. Based on these results, we selected "asr" as our primary submission for speech recognition track.

It the particular domain of non-native speech recognition, the usefulness of the phoneme language model seems to be minor, unlike on the $\mathrm{CV}$ and LS test sets in Table 2. However, this result could be unreliable because the IWSLT development set is very small.

We note that all proposed systems outperform publicly available Google and Microsoft ASR on all files in the development set, see the last two rows of Table 3.

\begin{tabular}{l|cccc} 
& AMIa & Teddy & Autocentrum & Audit \\
\hline asr & 8.87 & $\mathbf{5 . 2 0}$ & 15.94 & 22.40 \\
asr_lm $\dagger$ & 3.45 & 2.02 & 4.16 & 6.15 \\
asr_lm $\dagger^{*}$ & 5.30 & 4.33 & 8.64 & 18.16 \\
asr_slt & 9.77 & 4.35 & 16.40 & 22.91 \\
asr_slt_lm $\dagger$ & 3.45 & 2.21 & 4.00 & 6.54 \\
asr_slt_lm $\dagger^{*}$ & 5.34 & 4.20 & 6.92 & 20.07 \\
asr_bert & 10.22 & 3.99 & 13.38 & 24.76 \\
asr_lm $\ddagger$ & 10.79 & 4.36 & 17.24 & 25.09 \\
asr_slt_lm $\ddagger$ & $\mathbf{1 0 . 8 8}$ & 3.60 & $\mathbf{1 7 . 3 4}$ & $\mathbf{2 6 . 6 4}$ \\
\hline Gold & 34.95 & 45.57 & 36.56 & 38.97
\end{tabular}

Table 5: German BLEU scores on the IWSLT development set. $\dagger$ submitted without punctuation and segmentation. $\ddagger$ submitted with punctuation and segmentation after the deadline. * lower case BLEU.

\section{Punctuation, Truecasing and Segmentation}

Our ASR system produces lowercased, unpunctuated text, but the machine translation works on capitalized, punctuated text, segmented to individual sentences. We use the same biRNN punctuator, truecaser and segmenter as Macháček et al. (2020). The punctuator is a bidirectional recurrent neural network by Tilk and Alumäe (2016) trained on the English side of CzEng (Bojar et al., 2016). The truecaser uses tri-grams (Lita et al., 2003). We use a rule-based Moses Sentence Splitter (Koehn et al., 2007). More details are in Macháček et al. (2020), Section 4.2.

\section{Machine Translation}

Our submission to the SLT track relies on the MT systems, which are used also by ELITR project and are described in their submission to this task (Macháček et al., 2020). We do not rely on their validation for this task. As our primary MT systems, we select "WMT18 T2T" for Czech and "de T2T" for German, because they were easily accessible 


\begin{tabular}{l|ccc}
\multirow{2}{*}{ Name } & \multicolumn{2}{|c}{ Initialization } & \multirow{2}{*}{ LM rescoring } \\
& Encoder & Decoder & \\
\hline asr (primary) & random & random & no \\
asr_lm & random & random & yes \\
asr_slt & EN CS & CS EN & no \\
asr_slt_lm & EN CS & CS EN & yes \\
bert & EN CS & BERT & no
\end{tabular}

Table 6: Submitted English ASR configurations. "EN CS" means the Transformer encoder was initialized with the encoder weights from a translation model trained from English phonemes to Czech graphemes. "CS EN" means the decoder was initialized from an MT model translating Czech phonemes to English graphemes.

through Lindat service ${ }^{7}$.

"WMT18 T2T" was originally trained for English-Czech WMT18 news translation task (Popel, 2018), and was also between the top systems in WMT19 (Popel et al., 2019). It is a singlesentence Transformer Big model in Tensor2Tensor framework (Vaswani et al., 2018). "de T2T" is a similar system, but trained on the data for EnglishGerman WMT news translation. Tables 4 and 5 present BLEU scores of our primary systems for Czech and German, respectively. Note that the files Teddy, Autocentrum and Audit are very short.

We submit also all other machine translation systems for Czech and German by ELITR with our "asr" source for contrastive evaluation. See Macháček et al. (2020) for more details.

\section{Submission Summary}

We participate in two tracks of the non-native speech translation task: speech recognition, and speech translation into both Czech and German. In both cases, our submissions are off-line.

The acoustic model was initialized from a checkpoint trained on other data than allowed for the task. Therefore, our systems are unconstrained.

For the speech recognition track, we utilize our speech recognition pipeline in various configurations. We first obtain the phoneme transcripts using the acoustic model. For configurations marked with ".lm", we additionally use a phoneme language model during the acoustic model inference. Subsequently, we feed these phonetic transcripts to the phoneme-to-grapheme translation model. We have three variants of this model: plain ("asr"), with pre-trained weights from SLT ("slt"), and with pre-

\footnotetext{
${ }^{7}$ https://Iindat.mff.cuni.cz/services/ translation/
}

trained weights from SLT for encoder and BERT for decoder ("bert"). In this manner, we yield five different configurations for submission (see Table 6). The transcripts are then punctuated and truecased. Based on the punctuation, we further segment the transcripts. Our primary submission for the ASR track is the "asr" system.

We do not have our own translation model. To participate in the translation track, we utilize the MT systems of the ELITR project, which are mostly Transformer neural models. We select as our primary submission the "asr" system.

\section{Conclusion}

We presented our submissions to the Non-Native Speech Translation Task for IWSLT 2020.

For the non-native speech recognition, we proposed a pipeline that consists of an acoustic model and a phoneme-to-grapheme model. We demonstrated that the proposed pipeline surpasses commercially used ASR on the development set.

To participate in the non-native speech translation track, we use off-the-shelf translation model on our ASR transcripts.

\section{Acknowledgments}

The research was partially supported by the grants 19-26934X (NEUREM3) of the Czech Science Foundation, H2020-ICT-2018-2-825460 (ELITR) of the EU, 98120 of the Grant Agency of Charles University and by SVV project number 260575 .

\section{References}

Ebrahim Ansari, Amittai Axelrod, Nguyen Bach, Ondrej Bojar, Roldano Cattoni, Fahim Dalvi, Nadir Durrani, Marcello Federico, Christian Federmann, Jiatao Gu, Fei Huang, Kevin Knight, Xutai Ma, Ajay Nagesh, Matteo Negri, Jan Niehues, Juan Pino, Elizabeth Salesky, Xing Shi, Sebastian Stüker, Marco Turchi, and Changhan Wang. 2020. Findings of the IWSLT 2020 Evaluation Campaign. In Proceedings of the 17th International Conference on Spoken Language Translation (IWSLT 2020), Seattle, USA.

Willem D Basson and Marelie H Davel. 2013. Category-based phoneme-to-grapheme transliteration.

Ondřej Bojar, Ondřej Dušek, Tom Kocmi, Jindřich Libovický, Michal Novák, Martin Popel, Roman Sudarikov, and Dušan Variš. 2016. CzEng 1.6: Enlarged Czech-English Parallel Corpus with Processing Tools Dockered. In Text, Speech, and Dialogue: 19th International Conference, TSD 2016, number 
9924 in Lecture Notes in Computer Science, pages 231-238, Cham / Heidelberg / New York / Dordrecht / London. Masaryk University, Springer International Publishing.

B. Decadt, J. Duchateau, W. Daelemans, and P. Wambacq. 2001. Phoneme-to-grapheme conversion for out-of-vocabulary words in large vocabulary speech recognition. In IEEE Workshop on Automatic Speech Recognition and Understanding, 2001. ASRU '01., pages 413-416.

Michael Denkowski and Graham Neubig. 2017. Stronger baselines for trustable results in neural machine translation. In Proceedings of the First Workshop on Neural Machine Translation, pages 18-27.

Jacob Devlin, Ming-Wei Chang, Kenton Lee, and Kristina Toutanova. 2018. Bert: Pre-training of deep bidirectional transformers for language understanding. arXiv preprint arXiv: 1810.04805 .

Shuoyang Ding, Adithya Renduchintala, and Kevin Duh. 2019. A call for prudent choice of subword merge operations in neural machine translation. In Proceedings of Machine Translation Summit XVII Volume 1: Research Track, pages 204-213.

Rohit Gupta, Laurent Besacier, Marc Dymetman, and Matthias Gallé. 2019. Character-based nmt with transformer. arXiv preprint arXiv:1911.04997.

Axel Horndasch, Elmar Nöth, Anton Batliner, and Volker Warnke. 2006. Phoneme-to-grapheme mapping for spoken inquiries to the semantic web. In Ninth International Conference on Spoken Language Processing.

Oleksii Hrinchuk, Mariya Popova, and Boris Ginsburg. 2019. Correction of automatic speech recognition with transformer sequence-to-sequence model. arXiv preprint arXiv: 1910.10697.

Biing-Hwang Juang and Lawrence R Rabiner. 2005. Automatic speech recognition-a brief history of the technology development. Georgia Institute of Technology. Atlanta Rutgers University and the University of California. Santa Barbara, 1:67.

Philipp Koehn et al. 2007. Moses: Open Source Toolkit for Statistical Machine Translation. In $A C L$ Interactive Poster and Demonstration Sessions, page 177-180, USA. Association for Computational Linguistics.

Oleksii Kuchaiev, Jason Li, Huyen Nguyen, Oleksii Hrinchuk, Ryan Leary, Boris Ginsburg, Samuel Kriman, Stanislav Beliaev, Vitaly Lavrukhin, Jack Cook, Patrice Castonguay, Mariya Popova, Jocelyn Huang, and Jonathan M. Cohen. 2019. Nemo: a toolkit for building ai applications using neural modules.

Jason Li, Vitaly Lavrukhin, Boris Ginsburg, Ryan Leary, Oleksii Kuchaiev, Jonathan M. Cohen, Huyen Nguyen, and Ravi Teja Gadde. 2019. Jasper: An
End-to-End Convolutional Neural Acoustic Model. In Proc. Interspeech 2019, pages 71-75.

Lucian Vlad Lita, Abe Ittycheriah, Salim Roukos, and Nanda Kambhatla. 2003. Truecasing. In Proceedings of the 41st Annual Meeting on Association for Computational Linguistics - Volume 1, ACL '03, page 152-159, USA. Association for Computational Linguistics.

Dominik Macháček, Jonáš Kratochvíl, Sangeet Sagar, Matúš Žilinec, Ondřej Bojar, Thai-Son Nguyen, Felix Schneider, Philip Williams, and Yuekun Yao. 2020. ELITR Non-Native Speech Translation at IWSLT 2020. In Proceedings of the 17th International Conference on Spoken Language Translation (IWSLT 2020), Seattle, USA.

Vassil Panayotov, Guoguo Chen, Daniel Povey, and Sanjeev Khudanpur. 2015. Librispeech: an asr corpus based on public domain audio books. In 2015 IEEE International Conference on Acoustics, Speech and Signal Processing (ICASSP), pages 5206-5210. IEEE.

Thuong-Hai Pham, Dominik Macháček, and Ondřej Bojar. 2019. Promoting the knowledge of source syntax in transformer nmt is not needed. Computación y Sistemas, 23(3):923-934.

Peter Polák. 2020. Spoken language translation via phoneme representation of the source language. Master's thesis, Charles University.

Martin Popel. 2018. CUNI transformer neural MT system for WMT18. In Proceedings of the Third Conference on Machine Translation: Shared Task Papers, pages 482-487, Belgium, Brussels. Association for Computational Linguistics.

Martin Popel, Dominik Macháček, Michal Auersperger, Ondřej Bojar, and Pavel Pecina. 2019. English-czech systems in wmt19: Document-level transformer. In WMT.

Ivan Provilkov, Dmitrii Emelianenko, and Elena Voita. 2019. Bpe-dropout: Simple and effective subword regularization. arXiv preprint arXiv:1910.13267.

Michael D Riley and Andrej Ljolje. 1992. Recognizing phonemes vs. recognizing phones: a comparison. In Second International Conference on Spoken Language Processing.

Elizabeth Salesky, Matthias Sperber, and Alan W Black. 2019. Exploring phoneme-level speech representations for end-to-end speech translation. In Proceedings of the 57th Annual Meeting of the Association for Computational Linguistics, pages 18351841, Florence, Italy. Association for Computational Linguistics.

Rico Sennrich, Barry Haddow, and Alexandra Birch. 2016. Neural machine translation of rare words with subword units. In Proceedings of the 54th Annual Meeting of the Association for Computational 
Linguistics (Volume 1: Long Papers), pages 17151725.

Ottokar Tilk and Tanel Alumäe. 2016. Bidirectional recurrent neural network with attention mechanism for punctuation restoration. pages 3047-3051.

Ashish Vaswani, Samy Bengio, Eugene Brevdo, Francois Chollet, Aidan N. Gomez, Stephan Gouws, Llion Jones, Łukasz Kaiser, Nal Kalchbrenner, Niki Parmar, Ryan Sepassi, Noam Shazeer, and Jakob Uszkoreit. 2018. Tensor2tensor for neural machine translation. CoRR, abs/1803.07416.

Ashish Vaswani, Noam Shazeer, Niki Parmar, Jakob Uszkoreit, Llion Jones, Aidan N Gomez, Łukasz Kaiser, and Illia Polosukhin. 2017. Attention is all you need. In Advances in neural information processing systems, pages 5998-6008.

Alex Waibel, Toshiyuki Hanazawa, Geoffrey Hinton, Kiyohiro Shikano, and Kevin J Lang. 1989. Phoneme recognition using time-delay neural networks. IEEE transactions on acoustics, speech, and signal processing, 37(3):328-339. 